\title{
An Improved Routing Algorithm in OSPF for Energy Saving
}

\author{
Jinfan Tang ${ }^{1, a}$, Wei Fei ${ }^{2, b}$, Xueping Ren ${ }^{2, \mathrm{c}}$, Ming Jiang ${ }^{2, \mathrm{~d}}$ and Xianghua ${ }^{2, \mathrm{e}}$ \\ ${ }^{1}$ Information Engineering school, Hangzhou Dianzi Univiersity, Hangzhou, China, 310018 \\ ${ }^{2}$ Institute of Software and Intelligent Technology, HangZhou Dianzi University, \\ Hangzhou, China, 310018 \\ atangjf@hdu.edu.cn, b1103873905@qq.com, 'renxp@hdu.end.cn, djiangming@hdu.end.cn, \\ exhxu@hdu.end.cn
}

Keywords: OSPF; energy saving; routing protocol; energy consumption

\begin{abstract}
Along with the vigorous development of the Internet, the total energy consumption presents rapid growth trend, so energy saving and emission reduction of network industry is imminent. Many energy-saving strategies of the networks emphasize only a single aspect of the energy consumption while lack of a global view from the network or combination with other energy-saving schemes to study. Based on the analysis of the SPF (Shortest Path First) algorithm in OSPF (Open Shortest Path First) protocol and the energy margin of different types of routers between the idle state and the state to process the packet, we propose an energy-saving algorithm of routing protocol: Minimum Energy Consumption Routing (MECR) which considers the factor of energy consumption. By applying the algorithm, routers can select the minimal total of energy margin routing path among all equivalent paths. The experimental results show that the algorithm can save a part of energy consumption in the router which uses the MECR strategy by applying to different scale networks. Moreover, computational complexity of the algorithm is lower and it can be well compatible with the OSPF routing protocol.
\end{abstract}

\section{Introduction}

Nowadays, due to energy shortages and many non-renewable resources, the international community gradually focus on the concept of energy saving. The data statistics show that Ethernet access nodes across the global has more than 3 billion in 2010, and the number of switches, routers and other equipment which connect thereto are also enormous. The whole network system consists of IT equipments, such as the servers, routers, storage and switches etc. Acting as the hub of the Internet, the router connects to the various devices of the Internet. According to estimates released by the US Department of Energy Memorandum, the Internet-related electric quantity in America was about 36TW-h, and the router's consumption reached 5.2TW-h [1] in 1999.

With greatly concern about how to improve the effective utilization of energy and control the electricity of network systems, researchers have proposed a series of energy-saving mechanisms and measures. In the perspective of the network granularity, we can classify the existing energy-saving strategies into two categories: node-level strategies, network-level strategies [2].

Currently, there are many saving strategies that modify the network protocol have been proposed in the global network level, but each has its shortcomings. Moreover, most of network-level energy-saving solutions did not consider the energy consumption of the router. GTE strategy concentrated network traffic to a subset of multiple available paths for transmission [3]. However, it had high computational complexity, poor scalability. Based on the network topology and traffic matrix information, GNRE was a traffic loads-aware energy-saving strategy which iteratively deleted nodes and links from the topology. But it had some limitations on the compatibility and robustness [3]. Jones etc had some research of protocols in the field of energy saving in wireless networks [4]. On this basis, the descendants presented a novel integrated green routing algorithm and set energy consumption as weight between nodes. As a result, this algorithm could minimize energy consumption [5], but it was only for wireless networks. Moreover, the energy of the sensor node is limited, while the router node is not the case [6]. There are two typical 
node-level strategies: Equipment sleep technology solution and Dynamic adaptation technology solution, but the complexity of hardware implementation is large and the variability of network system can lead to the degradation of performance.

In this paper, we present a green routing algorithm for energy-saving strategy aimed at network level, named Minimum Energy Consumption Routing (MECR) strategy. The research is accomplished in three steps and the algorithm sets energy consumption as a second weight between nodes in wired network. At first, we summarize related work and discuss multi-constrained shortest path (MCSP) problem to select the optimal path. Secondly, we add a new parameter in routing algorithm in OSPF. The improved Dijkstra algorithm is proposed by describing MECR problem and presenting a mathematical programming formulation can minimize energy-consumption of transmission path.

The paper is organized as follows: Sec. II shows the related work of energy saving and multi-constrained shortest path (MCSP) problem; in Sec. III the protocol is proposed and the implementation of the MECR is given. The experimental results are shown in Sec. V. Finally, conclusion and the future research activities are discussed in Sec. VI.

\section{Preliminaries and Work Motivations}

In this paper, we focus on the study for the energy-saving technologies in the field of Internet cable network, especially for energy saving mechanisms of routing devices and routing protocol. A typical energy-efficient routing protocol scheme (G-OSPF: Green OSPF) is presented in [7], the EAR algorithm enables the router to shut down a part of links in the case of low load. The result shows more than $60 \%$ of the links in the actual Internet can be turned off. However, it will cause severe downturn of the network performance when the network load is heavy due to the factor of flow variability.

The energy consumption of basic router which unplugged all cables, line cards and other component was more than $50 \%$ of the total consumption. But the overall energy consumption would increase with the line card and the type of line card would also affect energy consumption, so different types of routers in the network have different energy consumption. A link includes light pre-amplifier, the repeater optical amplifier, and the light post-amplifier etc., and then the energy consumption of different types of links is also different [8]. Turning off the device is the most direct way of energy-saving when router equipment or component is in an idle state. Researchers followed a deep exploration of hibernate technology of routing devices, whereas the complexity of hardware implementation is large, and increases the storage and computation overhead $[9,10,11]$. In [12], Cianfrani indicated that the maximum variation in power use in the experiments is about 20 watts between the idle state of router and the scenarios with packet traffic. The difference extrapolated is between 150 and 200 with a fully loaded 12008 chassis. These effects are relatively unremarkable, but cannot be discounted.

In view of the above, we focus our attention on the network-level energy efficiency with taking into account the factor of energy consumption of router. The MECR strategy proposed in this paper has a lightweight modify to OSPF protocol and calculates the energy-saving routing path based on the router's power information regardless of the load variability. The new routing algorithm selects the shortest path of the minimum total energy margin of routers from multiple shortest paths which always exist in the network. The new algorithm can be thought as analogous to the Multi-constrained shortest path algorithm proposed in [13-15]. The complexity of finding out all of the shortest paths from the graph is NP-complete, so it will be impossible to get a good algorithm by comparing the size of the total energy margin of these shortest paths [16]. Then we propose a heuristic algorithm for optimal path based on Dijkstra algorithm in this paper.

The main advantage of this solution is the topology does not change and the alteration of shortest path does not affect the performance of network system. Of course, comparing with other energy-saving strategies, the energy-saving effect of MECR is not significant. But from a macro point of view and long-term development of the Internet, this solution will save energy quite a lot. 


\section{Protocol design}

Description of proposed OSPF routing protocol.Based on the memo documents version 2 of the OSPF protocol [17], we improve the OSPF routing protocol. In this section, we will detail the proposed algorithm for OSPF. We define the link weight according to the power margin of router which is introduced in the improved algorithm. In view of the above, we propose formula (1) to denote the power margin of a router between the idle state and the state to process the packet.

$$
P_{m}(X)=P_{t}(X)-P_{i}(X)
$$

where the vector $X$ defines the router type, $\mathrm{P}_{\mathrm{t}}$ is the power consumption of the state with packet traffic, and $\mathrm{P}_{\mathrm{i}}$ denote the idle state.

We propose the simplified model of an OSPF router (as shown in Fig. 1), where the functions to be implemented are shown with gray box [18]. The figure shows that the interface information of OSPF router will be modified and the Energy-saving Dijkstra algorithm will be used to path computation. OSPF is a link state routing protocol, using Dijkstra algorithm to calculate the shortest path. Dijkstra's algorithm in OSPF routing protocol is implemented by the priority queue. The protected members in PriQElt class (classes representing an individual element on a Priority queue) define the sum of the link cost (cost0) and arbiter (cost1) [19]. Because the proposed algorithm is used to select the path of the least total energy consumption, it needs to define a variable (cost2) to store energy consumption value. We define a parameter of forward cost 2 in Link class to calculate the new cost.

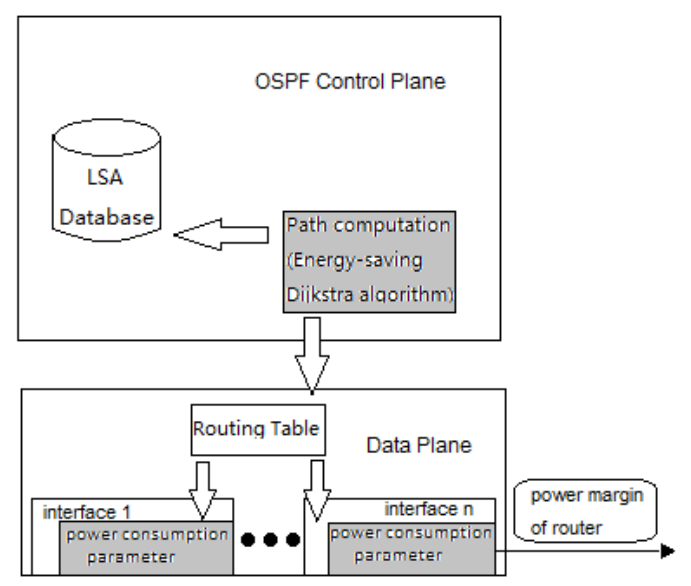

Fig. 1: OSPF green router

We describe the proposed OSPF as follows:

Step 1: Define a variable representing the energy consumption in the priority queue class.

Step 2: Add 1_fwdcst2 to the Link class of LSA (Link state advertisement is an OPSF packet, which contains shared routing information and link state) header file, it is used to store a link's forward cost 2 .

Step 3: Improve the shortest path algorithm in OSPF.

Algorithm implementation.In this paper, the proposed Algorithm is to find the least of total energy path from multiple shortest paths which can be regarded as the multi-constrained shortest path (MCSP) problem, called Minimum Energy Consumption Routing (MECR) problem. MECR problem is abstracted as a mathematical model which link is modeled by cost and power, where power is the power margin of a router between the idle state and the state to process the packet calculated by formula (1). The network is modeled as a directed graph $\mathrm{G}=(\mathrm{V}, \mathrm{E})$, where $\mathrm{V}$ is the set of nodes, and $E \subseteq V \times V$ is the set of links. For each link $1 \in \mathrm{E}$, we assume $c(l)$ is the cost of link and let $f(l)$ denote the additive cost of the link $1, \mathrm{~s} \in \mathrm{V}$ is the source node and $\mathrm{t} \in \mathrm{V}$ is the destination node. The mathematical programming formulation of the LPC problem is given by [20]:

Finding a path from $s$ to $t$ in $G$ 
$c(\widetilde{p})=\min _{p \in P} \sum_{l \in P} c(l)$

subject to $f(\tilde{p})=\min _{p \in P} \sum_{l \in P} f(l)$

where $\mathrm{P}$ is the set of all paths from to $\mathrm{s}$ to $\mathrm{t}$.

The procedure of algorithm is as follows:

1) Initialize algorithm.

2) Let $\mathrm{V}$ be the new node just joining the shortest path tree, then scan its neighbor nodes and join the candidate list. Let $\mathrm{W}$ be the neighbor node.

3) If node $\mathrm{W}$ is already on the shortest-path tree \{

Examine the next link;

Calculate a new distance value new_cost $=\mathrm{V} \rightarrow \cos t 0+\mathrm{tlp} \rightarrow 1$ _fwdest (Forward cost 0$)$;

Calculate a new energy consumption value new_cost $2=\bar{V} \rightarrow \operatorname{cost} 2+$ tlp $\rightarrow 1$ fwdcst 2

(Forward cost2);

\}

4) If node $W$ is already in the candidate list \{

If new_cost is greater than the value of cost 0 of $\mathrm{W}$ in the candidate list,

Continue to examine the next link;

Else if it is less than the value of cost 0 of the candidate node $\mathrm{W}$ in the list, Else \{ Delete the node W;

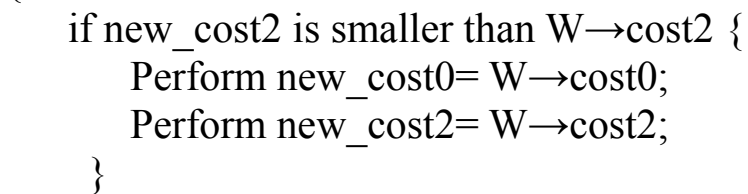

Else use proclaimed link directly to calculate the next hop; \}

\}

5) If node $\mathrm{W}$ is not in the candidate list or new_cost is less than $\mathrm{W} \rightarrow \operatorname{cost} 0\{$

Join $\mathrm{W}$ to the candidate list;

Use the cost of link to calculate the next hop;

Set the next hop value of W; \}

6) Repeat steps second to fifth, until the candidate list is empty. The Shortest Path of least energy consumption is saved in the shortest path tree.

\section{Experiments and Analysis}

In this paper, we implement the proposed algorithm and do the experiments using Visual $\mathrm{C}++$ 6 in Windows 7. We use the famous American backbone network - NSFNet network as the network topology (as shown in Fig. 2), which contains 14 router nodes and 19 links. In this topology, every link has two metrics. In order to enable the network closer to the actual network topology, we choose the high popularity of various series routers of Cisco brand and select 14 different routers from them to build NSFNet network topology. According to formula (1), we can obtain the additive cost of a link. The specific data of this case are shown in Table 1. For example, the energy margin of Cisco 2811 is $20 \mathrm{~W}$. The energy saving ratio is hard to be computed under heavy network load, and during this period the effect of energy saving is poor due to most of routers have been in operation state. We assume that the experiments are done under the network environment of low network load. In order to reflect the energy-saving effect directly, a set of data flow will transfer from the first router to the 14th router without traffic through other routers. 


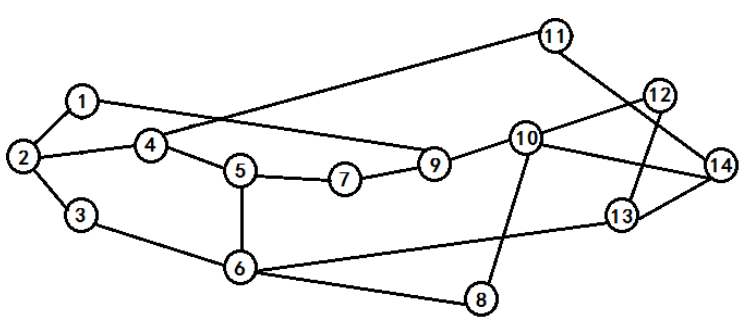

Fig. 2: NSFNET network

We do a lot of simulation experiments by using different combination of routers to obtain some result data. To calculate the energy saving rate, we must compare with the total power margin of all routers on shortest path produced in OSPF routing protocol originally. There may be only one shortest path in actual network, but in general it will be equal cost multi-path to achieve load balancing in large network, and it also means that there are a number of shortest paths in somewhat complicated networks. In order to better explanation, there are four shortest paths in NSFNet network. The topology in this example has four shortest paths (as shown in Fig. 3(a)), marked in red, green, blue and yellow respectively. The new algorithm eventually gets the path $(1 \rightarrow 2 \rightarrow 3 \rightarrow 6$ $\rightarrow 13 \rightarrow 14$ ) (as shown in Fig. 3(b)) with the least sum of energy consumption margin among the four paths. When we use Dijkstra algorithm in original OSPF protocol to calculate, the path $(1 \rightarrow 2$ $\rightarrow 4 \rightarrow 11 \rightarrow 14$ ) is selected.

Table 1: Cisco routers and margin value

\begin{tabular}{|c|c|c|c|c|c|c|c|}
\hline ID & 1 & 2 & 3 & 4 & 5 & 6 & 7 \\
\hline Full & Cisco 2811 & Cisco & Cisco & Cisco & Cisco & Cisco & Cisco \\
\hline name & & 7204VXR & 1841 & 2851 & 3845-SEC/K9 & 7507 & 3925 \\
\hline Energy margin & $20 \mathrm{~W}$ & $20 \mathrm{~W}$ & $5 \mathrm{~W}$ & $50 \mathrm{~W}$ & $60 \mathrm{~W}$ & $30 \mathrm{~W}$ & $60 \mathrm{~W}$ \\
\hline ID & 8 & 9 & 10 & 11 & 12 & 13 & 14 \\
\hline \multirow[t]{2}{*}{ Full name } & Cisco GSR & Cisco 3945 & Cisco & Cisco & Cisco 890 & Cisco & Cisco \\
\hline & 12008 & & 7201 & 3945 & & $4451-\mathrm{x}$ & 7000 \\
\hline Energy margin & $60 \mathrm{~W}$ & $80 \mathrm{~W}$ & $20 \mathrm{~W}$ & $80 \mathrm{~W}$ & $10 \mathrm{~W}$ & $60 \mathrm{~W}$ & $100 \mathrm{~W}$ \\
\hline
\end{tabular}

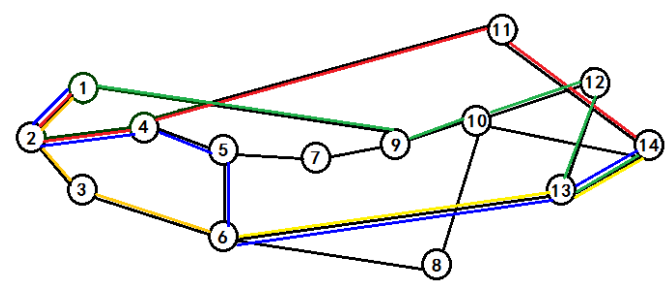

(a)

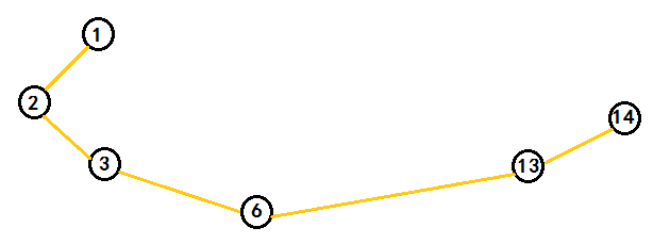

(b)

Fig. 3: (a) The four shortest path (b) The Shortest Path of least energy consumption We use the following formula to calculate the total energy consumption firstly:

$$
\text { Total_ } P=\sum_{i=1}^{N} P\left(x_{i}\right)
$$

Total_P represents the sum of the power margin of all routers which are on the shortest path in the proposed algorithm; $\mathrm{P}\left(\mathrm{X}_{\mathrm{i}}\right)$ denotes energy margin of router $\mathrm{X}_{\mathrm{i}}$. Secondly, ES_rate is the rate of energy saving, and Total_P1 is the total margin value which results from the original algorithm, Total_P2 returns the value of total energy margin which uses the proposed algorithm.

$$
E S \_r a t e=(\text { Total_P1-Total_P2 }) / \text { Total_P1 }
$$

From the above experiment, the data (as shown in Tab. 2) are obtained by using formula (4) and ES_rate is calculated by using formula (5). We find that Total_P1 is 290W and Total P2 is $235 \mathrm{~W}$, then we conclude that the algorithm can save about $19 \%$ of energy consumption in this case. 
Table 2: Margin value of each path

\begin{tabular}{|c|c|}
\hline Routing path & Total energy margin of path \\
\hline $1 \rightarrow 2 \rightarrow 4 \rightarrow 11 \rightarrow 14$ & $290 \mathrm{~W}$ \\
\hline $1 \rightarrow 2 \rightarrow 3 \rightarrow 6 \rightarrow 13 \rightarrow 14$ & $235 \mathrm{~W}$ \\
\hline $1 \rightarrow 9 \rightarrow 10 \rightarrow 12 \rightarrow 13 \rightarrow 14$ & $280 \mathrm{~W}$ \\
\hline $1 \rightarrow 4 \rightarrow 5 \rightarrow 6 \rightarrow 13 \rightarrow 14$ & $320 \mathrm{~W}$ \\
\hline
\end{tabular}

We expand the scale of the network topology in order to obtain a more clear result. When increasing the number of routers gradually up to 100 on the basis of NSFnet network, we obtain the following trend graph of energy saving effects (as shown in Fig. 6). The figure shows that the lowest rate of energy savings is $7 \%$, and the highest rate reach $55 \%$. However, with the larger network size, energy savings also increases based on the data trend line in Fig 4. Finally we come to the conclusion that this algorithm can save considerable power consumption in the real network compare with the previous consumption.

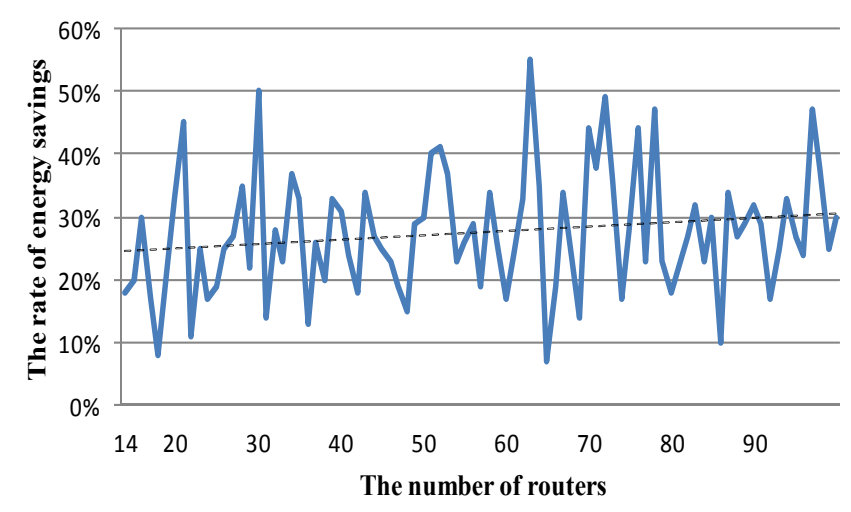

Fig. 4: Energy savings effects of different scale networks

\section{Conclusions}

At present, there have come many achievements on network energy-saving research. Energy saving schemes of network level fit in with the long-term development of the Internet in the future. Based on router energy consumption model, we obtain the energy consumption values of different type of the router. We propose an energy-saving algorithm of routing protocol. The example and experiments prove our algorithm is effective. From the experiment results, we can see that the algorithm saves energy consumption without changing the network device or the internal components. By applying to different scale networks, the algorithm can demonstrate the good effect of energy conservation during low traffic hours. Moreover, computational complexity of the algorithm is lower and can be well compatible with the OSPF routing protocol. Further study is combining the algorithm in this paper with other energy-saving mechanisms for greater effects such as EAR algorithm in G-OSPF.

\section{Acknowledgements}

Scientific research fund of Zhejiang Provincial education department of China (NO. Y201432390), Zhejiang Provincial key science and technology innovation team of China (NO. 2013TD20), National Science and Technology Support Program of China (No. 2014BAH23F01-2), National 863 plan project of China (NO. 2015AA011901), National High Technology Development program of China (NO.2015AA016102), Science and technology project of Zhejiang Province (No. 2014C01051). 


\section{References}

[1] ZHANG Fa et al. “Network Energy Consumption Models and Energy Efficient Algorithms” , Chinese Journal of Computers, 2012, 35(3): 603-615.

[2] LIN Chuang, TIAN Yuan, YAO Min, “Green Network and Green Evaluation: Mechanism, Modeling and Evaluation” , Chinese Journal of Computers, 2011, 34(4): 593-612.

[3] SHANG Yun-fei, XU Ming-wei, LI Dan, "Research on Energy-Saving Routing Devices and Protocols in the Internet” , Acta Electronica Sinica, China, November 2012.

[4] C. Jones, M. Sivalingam, P. Agrawal, and J. Chen, "A Survey of Energy Efficient Network Protocols for Wireless Networks”, Wireless Networks, July 2001.

[5] LIU Yan-jun, GUO Ai-huang, FENG Sheng-yi, "Research on Power Efficient Routing Algorithm in Green Optical Networks” , Acta Optica Sinica, China, April 2012.

[6] Feng Luo, Chunxiao Jiang, Haijun Zhang, "Node Energy Consumption Analysis in Wireless Sensor Networks”, 2014 IEEE 80 ${ }^{\text {th }}$ Vehicular Technology Conference, September 2014.

[7] A. Cianfrani, V. Eramo, M. Listanti, M. Marazza, “An Energy Saving Routing Algorithm for a Green OSPF Protocol” , IEEE INFOCOM 2010, San Diego (USA), March 2010.

[8] Zhang J, Wang X, “An Energy-Saving QoS Routing Protocol in Green Internet” , Cyberspace Technology, International Conference on. IET, 2013:88-93.

[9] Gupta M, Singh S, “Using low-power modes for energy conservation in ethernet LANs” , Anchorage: IEEE Press, 2007. 2451-2455.

[10] Gunaratne C, Christensen K, Nordman B, "Managing energy consumption costs in desktop PCs and LAN switches with proxying, split TCP connections, and scaling of link speed” , International Journal of Network Management, 2005, 15(5) : 297-310.

[11] Nedevschi S, Chandrashekar J, "Skilled in the art of being idle: reducing energy waste in networked systems” , In Proc of NSDI' 09. Boston: USENIX Association, 2009: 381-394.

[12] CHABAREK J, SOMEERS J, BAFORD P et al. "Power Awareness in Network Design and Routing” , The 27th IEEE Conference on Computer Communications, USA， 2008: 457-465.

[13] HU Yao-min, LIU Wei-ming, “Multi- constrained shortest path model and solving” , Journal of Hunan University of Science \& Technology(Natural Science Edition), China, 2010, 25(1):87-90

[14] Qiang S, Zong-yuan Y, “A New Algorithm for Vertices-constrained Shortest Path”, Computer Engineering, 2002.

[15] Jia Z, Varaiya P, "Heuristic methods for delay constrained least cost routing using k-shortest-paths” , IEEE Transactions on Automatic Control, 2006, (4): 707-712.

[16] Zhou Jinglun, Wu Huangqun, "Vertices-constrained Shortest Path Problems and Algorithm” , Systems Engineering, China, 1996, 14(5): 37-43.

[17] J. Mo, RFC2328_OSPF Version 2. http: //www.ietf.org/rfc/rfc2328.txt. 1998

[18] Cianfrani A, Eramo V, Listanti M, et al. “An OSPF-Integrated Routing Strategy for QoS-Aware Energy Saving in IP Backbone Networks" , in School of Information and Engineering, Henan Institute of Science and Technology. His, 2012, 9(3): 254-267.

[19] John T. Moy, OSPF Complete Implementation, America: Addison-Wesley Educational Publishers Inc, 2000

[20] Amaldi E, Capone A, G. Gianoli L, et al. "Energy management in IP traffic engineering with Shortest Path routing”, World of Wireless, Mobile and Multimedia Networks (WoWMoM), 2011 IEEE International Symposium on a. IEEE, 2011: 1-6. 